\title{
OPTIMAL PRODUCTS' HAND-HANDLE INTERFACE PARAMETER IDENTIFICATION
}

\author{
Harih, G.; Borovinsek, M.; Ren, Z. \& Dolsak, B. \\ Faculty of Mechanical Engineering, University of Maribor, Smetanova 17, SI-2000 Maribor, Slovenia \\ E-Mail: gregor.harih@um.si
}

\begin{abstract}
The paper reports findings of investigating optimal interface material parameters of product handle to improve its ergonomics by increasing the user performance, comfort, and lower the risk of cumulative trauma disorder development. Optimisation methods in combination with the finite element computer simulations of a human fingertip whilst grasping a handle were used to determine an interface foam material with optimal material properties for best mechanical behaviour of the system. A single objective function was defined to determine best material parameters of the interface foam material, which has to remain firm during low grasping forces to provide stability of the product grasp and appropriately deforms when a critical contact pressure is reached to provide higher contact area. This increases comfort and lowers the contact pressure on the hand and thereby the risk of injury development. The optimisation process yielded the optimised foam thickness and its stress-strain relationship for simulated contact of fingertip model grasping a product handle.

(Received in November 2014, accepted in March 2015. This paper was with the authors 1 month for 1 revision.)
\end{abstract}

Key Words: Ergonomics, Product Development, Foam Material, Computer Simulations, Finite Element Method, Optimisation

\section{INTRODUCTION}

The human hand is one of the most sophisticated and complex tools of a human. One of the key functions of the hand is the interaction with the physical environment, where the most important is the prehensile hand grasp with a physical object. In this way, it is effectively used as a tool for work, as well as the interface to use various powered and non-powered products, hand tools and equipment. Correct design of handheld products is crucial for preventing upper extremity acute trauma disorders (ATD) and cumulative trauma disorders (CTD), such as blisters, carpal tunnel syndrome, hand-arm vibration syndrome, tendonitis, etc. [1-5]. It has been shown that CTD account for about one third of the sick leave of workers, which results in high worker's compensations claims [5]. Therefore ergonomic principles should be included in the phase of industrial/mechanical product design, since the main function of the product and the form of the product are usually strongly connected [6-8]. The researchers have so far provided different guidelines and mathematical models for determining the sizes and shapes of the product handles and product grasping surfaces to improve ergonomics and therefore safety of the products and to prevent ATD and CTD [9-12].

Since skin is in direct contact with the products' interface material, its material properties are important in the grasping tasks. Therefore mechanical properties of skin and subcutaneous tissue have been extensively investigated by different researchers [13-19]. Both skin and subcutaneous tissue show non-linear viscoelastic properties, where the skin is stiffer than the subcutaneous tissue. Both have low stiffness regions at small strains followed by a substantial increase in the stiffness when the strain increases.

It has been shown that products which require high grip, push, pull or torque exertion on the handle produce high contact pressures, which is known to be one of the primary factors for the development of ATD and also CTD [4, 20-23]. Therefore many researchers have tried to propose maximum allowable contact pressures, which should not be exceeded. They have 
used different criteria for the maximum allowable contact pressures, therefore also the values differ [24-26]. Brand and Hollister provided rough guidelines for the maximum suggested pressure versus time application over bony prominences [24]. Aldien et al. provided guidelines of pressure discomfort (PDT) and pressure-pain threshold (PPT), where PPT is higher than PDT and values differ by the area of the hand [25]. Also different subjects reported different values due to subjective perception of the load on the hand. The PDT limit of $188 \mathrm{kPa}$ has been suggested by Aldien et al. [25], however Fransson-Hall and Kilbom [26] estimated its value at $104 \mathrm{kPa}$. Therefore the grasping surfaces should be designed to distribute contact pressure more evenly where the PDT limits are not exceeded to maintain the desired user performance [25]. A limit contact pressure on the fingertip $p_{\text {lim }}=100 \mathrm{kPa}$ was selected for the purpose of this investigation.

Research was usually focused on the sizes and the shapes of the product grasping surfaces, but neglected the interface materials, which are in direct contact with the user's hand. Fellows and Freivalds investigated suitability of foam materials for the interface materials to lower the contact pressure [27]. The test subjects reported increase in comfort rating and decrease in stability of the product grasp, which can be attributed to the foam deformation while grasping. It is necessary that the correct product's interface material determination considers also the mechanical behaviour of the skin and soft tissue while grasping.

The finite element (FE) method is very often used to successfully model, compare and evaluate material choice in the design phase of a product [28]. Deformations and stresses that represent basic results of the structural analysis using FE method are also an important aspect in the field of ergonomics. It has already been shown that cellular materials can be characterised to meet specific mechanical behaviour [29]. Within such context a composite hyper-elastic foam material that can lower the contact pressure whilst keeping the low deformation rate of the product handle material to maintain a sufficient stability rate when using the product was proposed [30]. Due to high complexity of the simulated system of fingertip and hyper-elastic foam with non-linear material behaviour, it is difficult to directly propose a foam material with optimal material properties for best mechanical behaviour of the system.

Therefore optimisation methods for material parameter identification of the interface hyper-elastic foam material were utilized in this paper. The optimisation was set to determine material parameters of the interface material which remains firm during low grasping forces to provide stability of the product in hands and deforms when a critical contact pressure is reached to provide higher contact area and thereby increase comfort and lower the risk of ATD and CTD development.

\section{THE METHODS}

\subsection{The finite element model - geometrical and boundary conditions}

A modern finite element simulation software Abaqus/CAE 6.10 from Dassault Systems (France) was used [31]. The used computational model of a fingertip and interface foam material was two-dimensional and symmetrical to decrease the required computational power for optimisation process. The FE model was based on previously developed fingertip FE models [32]. The product interface material has been modelled as a flat rectangle with two sections using $1 \mathrm{~mm}$ protection layer of EPDM rubber and a discretely scalable (from $2 \mathrm{~mm}$ to $6 \mathrm{~mm}$ ) hyper-elastic foam layer (Fig. 1).

The displacements and rotations of the rectangle representing the interface material of the product were fixed on the lower boundary. The displacement and rotations of the fingertip 
were fixed, except for the displacement along the vertical axis. In order to simulate grasping, fingertip displacement of $u_{p}=5 \mathrm{~mm}$ at the vertical top point of the finger bone was applied and directed normal to the interface material surface. The fingertip and the product interface material were meshed using a structured mesh of 5149 and 1920 CPE8 elements, respectively. CPE8 elements are eight node plane strain element for general purpose where displacement in $\mathrm{z}$-axis is zero. These elements allow reasonable simplification of simulation cases where the sections in z-axis do not vary too much.

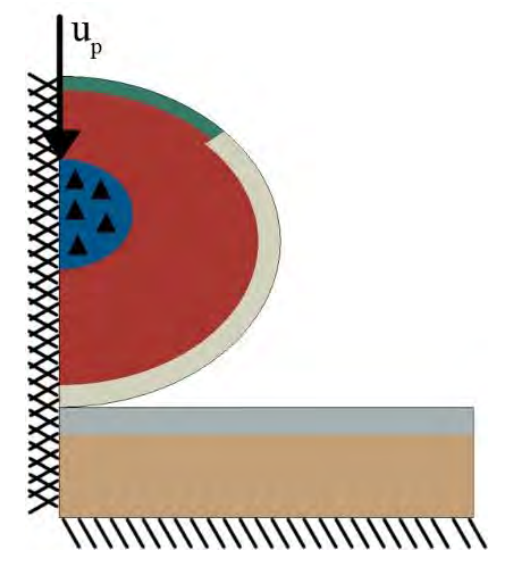

Figure 1: Geometrical and boundary conditions of the computational model.

\subsection{The finite element model - material properties}

The fingertip bone and nail were assumed to be linear elastic and isotropic with Young's modulus of $17 \mathrm{GPa}$ and $170 \mathrm{MPa}$, respectively, and a Poisson's ratio of 0.3 [33]. The material parameters of skin and subcutaneous tissue were defined using the Ogden hyper-elastic material model [33]. Since skin and subcutaneous tissue are almost incompressible, the Poisson ratio was defined as 0.4 [33].

Material parameters of the outer protective layer made of EPDM rubber were based on literature and simulated using Ogden hyper-elastic material model [30]. The material parameters used equalled $\mu_{1}=-4.989, \alpha_{1}=-1.928 \cdot 10^{-2}, \mu_{2}=0.1439, \alpha_{2}=6.207, \mu_{3}=5.752$, $\alpha_{3}=-0.4778, D_{1}=0.2282$. The material properties of the hyper-elastic foam (interface material) were not defined in advance but determined with the optimisation procedure.

\subsection{Optimisation procedure}

A single-objective optimisation problem was formulated to identify the interface hyper-elastic foam material with optimal material properties and its thickness based on the presented FE model. The occurrence of more than one local optima of the objective function was expected due to the usage of nonlinear material models and large deformation formulation.

The discretization of the geometry and contacts in the model cause the occurrence of numerical noise in the objective function thus a non-gradient based optimisation method i.e. a genetic algorithm with elitism and selection based on the biased roulette wheel scheme was chosen for the identification of material parameters. The genetic algorithm is a well-known meta-heuristic algorithm, which follows the natural evolution process, and can be used in various technical applications [34]. The inputs of the algorithm are control parameters, design variables and an objective function. The control parameters define the population size (number of individuals), the number of generations and the rate of crossover and mutation operations. The design variables were the material parameters of foam material model and its thickness. The applied objective function is described in the following. 
A limit contact pressure on the fingertip should not be exceeded for longer periods of time to prevent disorders. But if the limit pressure is reached and exceeded during the product usage, the pressure should rise as slow as possible in regard to the fingertip displacement. However, larger deformations of the product handle interface material decrease the users' product stability and therefore its control. So the product handle deformations should be as small as possible in the working regime of the contact pressures but when the limit contact pressure is reached and exceeded the pressure should rise as slowly as possible.

The intersection point $\mathrm{P}$ of the contact pressure curve with the limit contact pressure is depicted in Fig. 2. The fingertip displacement at this point is marked as $u_{\text {lim }}$ and the slope of the contact pressure at that point was computed as the first derivative $p$ ' of the contact pressure with regard to the fingertip displacement $p^{\prime}=(d p / d u)_{P}$.

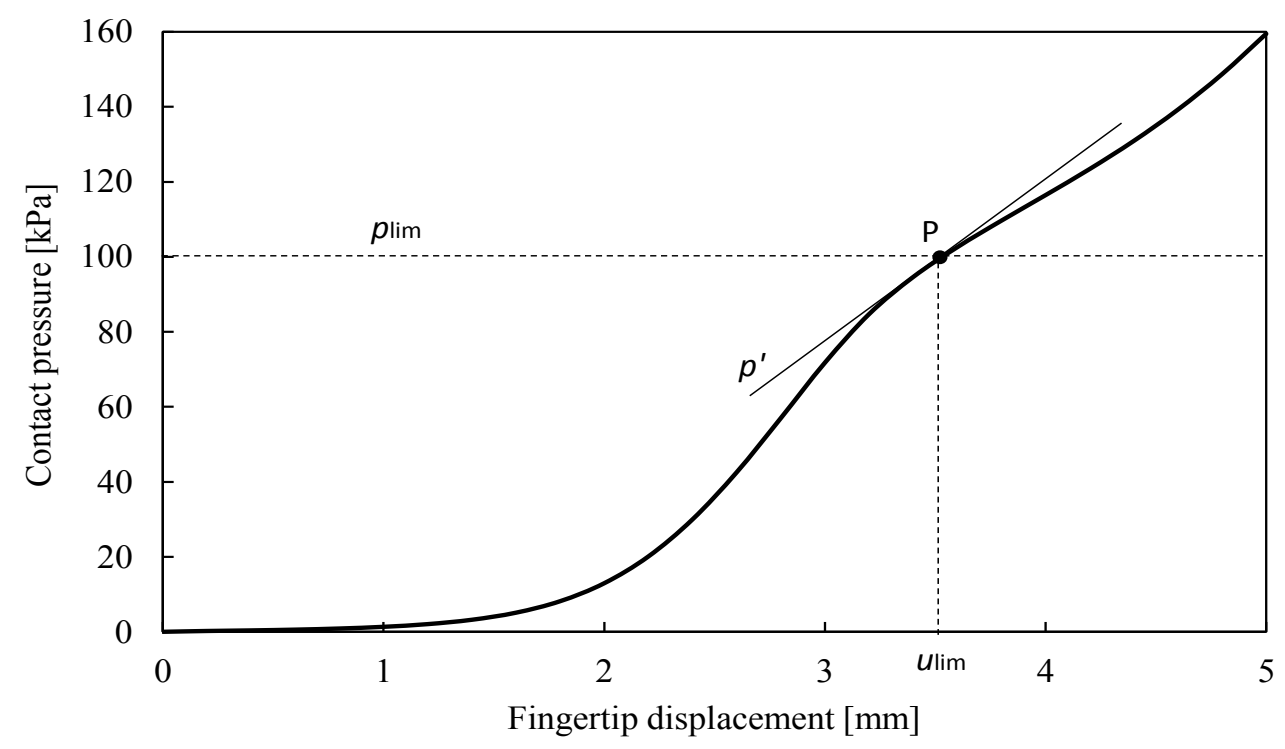

Figure 2: Typical contact pressure vs fingertip displacement curve.

Based on these observations the following single-objective function was formulated:

$$
f(\boldsymbol{x})=w_{1} \cdot\left(u_{\text {lim }}-u_{\text {steel }}\right)+w_{2} \cdot\left(\frac{\mathrm{d} p}{\mathrm{~d} u}\right)_{P}=u_{n}+p_{n}^{\prime}
$$

It comprises the fingertip displacement $u_{\text {lim }}$ and the slope of the contact pressure at the intersection point $\mathrm{P}$ where $\boldsymbol{x}$ represents the design variables and $w$ represents weights of individual parts of the optimization function.

The weights were determined with preliminary simulations in such a way that the values of both objective function parts were approximately in the interval between 0 and 1 . They were set to $w_{1}=1 / 3$ and $w_{2}=1 / 100$. The fingertip displacement $u_{\text {lim }}$ was beforehand reduced with a fingertip displacement value in a case of a steel tools handle which approximately equals $u_{\text {steel }}=3 \mathrm{~mm}$. In this way values for the normalized displacement $u_{n}$ and normalized pressure slope $p_{n}{ }_{n}$ were obtained.

The design variables $\boldsymbol{x}$ were the material parameters of the foam material and its thickness $h$. The foam material was modelled with a hyper-foam constitutive model defined with uniaxial test data [31]. The stress-strain test data was approximated with a three-linear curve to reduce the number of design variables (Fig. 3). The three-linear curve is determined with four data points $T_{i}$ where their coordinates correspond to the strain-stress pairs $\varepsilon_{i}, \sigma_{i}$. The first data point $\mathrm{T}_{1}$ was fixed at $(0,0)$ while the strain value of the fourth data point $\mathrm{T}_{4}$ was fixed at strain $\varepsilon_{4}=0.9$, since preliminary simulations showed maximum strains of 0.7 . All five other coordinates were taken as design variables with values within interval bounds given in Table 
I. The interval bound values were determined with a design of experiments procedure in order to find the unbound minimum of the described problem. The thickness of the foam material layer $h$ was varied from 2 to $6 \mathrm{~mm}$.

Table I: The design space of the design variables.

\begin{tabular}{|c|c|c|}
\hline Design variable & Lower bound & Upper bound \\
\hline$\varepsilon_{2}[/]$ & 0.01 & 0.08 \\
\hline$\varepsilon_{3}[/]$ & 0.11 & 0.78 \\
\hline$\sigma_{2}[\mathrm{kPa}]$ & 10 & 200 \\
\hline$\sigma_{3}[\mathrm{kPa}]$ & 20 & 400 \\
\hline$\sigma_{4}[\mathrm{kPa}]$ & 420 & 1400 \\
\hline$h[\mathrm{~mm}]$ & 2 & 6 \\
\hline
\end{tabular}

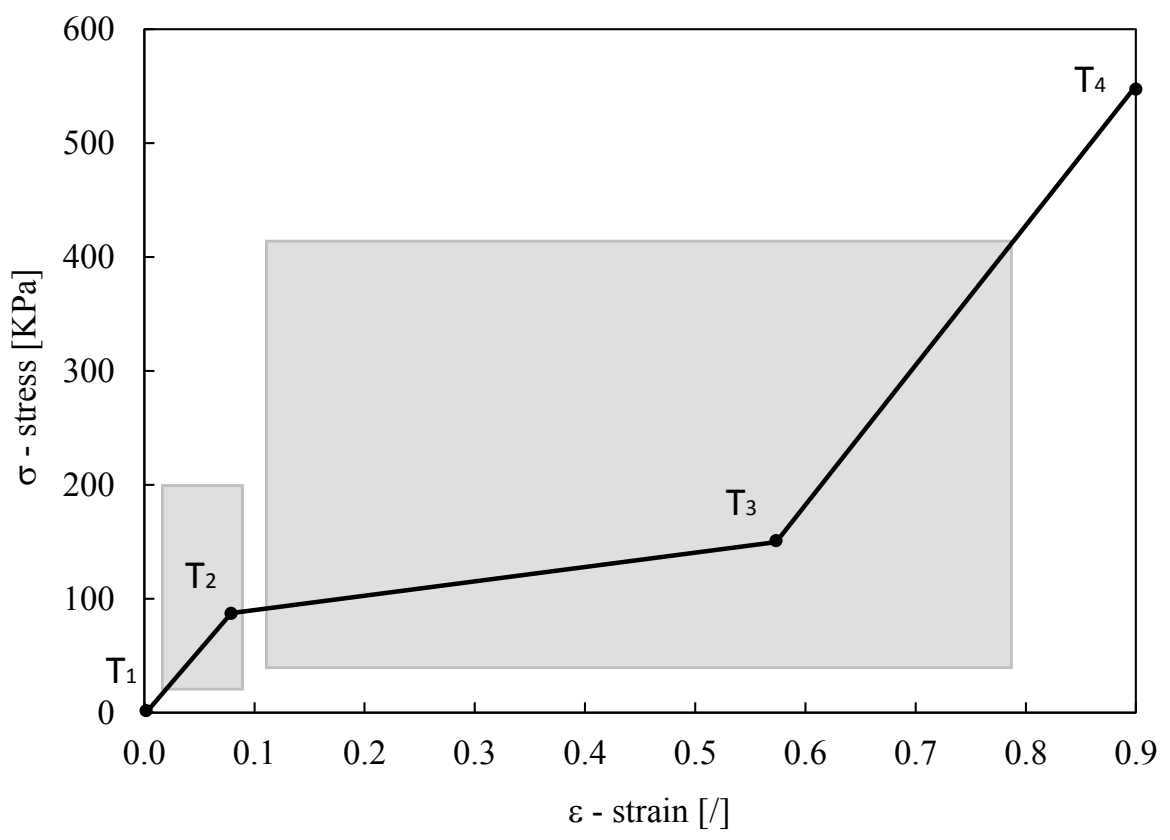

Figure 3: Foam material design space based on the uni-axial test data.

The optimization workflow for a single solution was divided into three consecutive steps. In the first step the current design variables are used to change the base FE simulation input file. Since the FE input file is a text file the change of material parameters is trivial, whereas the change in the interface foam layer thickness requires a change of the finite element mesh. The foam layer thickness was changed by uni-axial scaling of the interface foam layer nodes using a linear scaling algorithm. The second workflow step is the computer simulation and extraction of 26 contact pressure versus fingertip displacement data points which form the response curve depicted in Fig. 2. In the third step this data is used to determine the response curve intersection with the $100 \mathrm{kPa}$ line using a cubic spline interpolation of the response curve data points, where the fingertip displacement $u_{\text {lim }}$ and the contact pressure slope $p^{\prime}$ are determined and used to evaluate the optimization function value.

The genetic algorithm was run using 100 samples in a generation for 50 generations. The crossover parameter was set to 0.80 and the mutation parameter to 0.05 [35]. A personal computer was used to run the optimisation procedure in parallel and a high performance computer cluster was used for computational simulations. Each simulation was run on 4 CPUcores resulting in execution time of about one minute. 5000 simulations were run in total to find the optimal result. 


\section{THE RESULTS}

The 2D FE fingertip model has been verified and validated in our previous research in regard to existing FE models and experimental data [30].

The optimisation procedure was run for 50 generations. From the convergence curve can be seen that the best individual in a generation was constantly improved in the first thirty generations and then lastly changed in the $36^{\text {th }}$ generation. All solutions of the singleobjective genetic algorithm are shown in Figs. 4, 5 and 6. They are depicted in as normalized displacement versus normalized pressure slope.

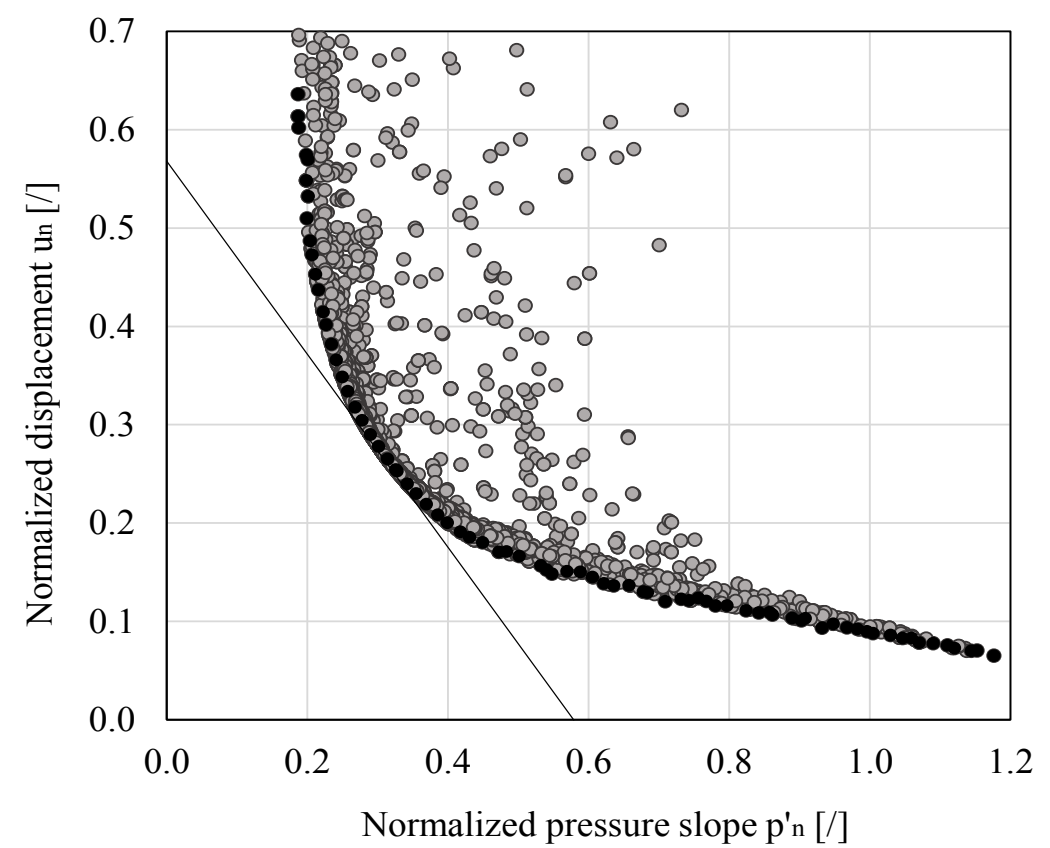

Figure 4: The solutions of the optimization problem (grey) with their Pareto front (black).

Fig. 5 shows all solutions of the optimization procedure in terms of optimization function value versus interface foam layer thickness in two different scales. The optimization function value ranges from an optimum value of 0.579 to the largest value of 1.27 , while the interface layer thickness ranges from 2.07 to $5.99 \mathrm{~mm}$ (left side of Fig. 5). Single global minima can be observed at the interface foam thickness of $4.83 \mathrm{~mm}$ (right side of Fig. 5).
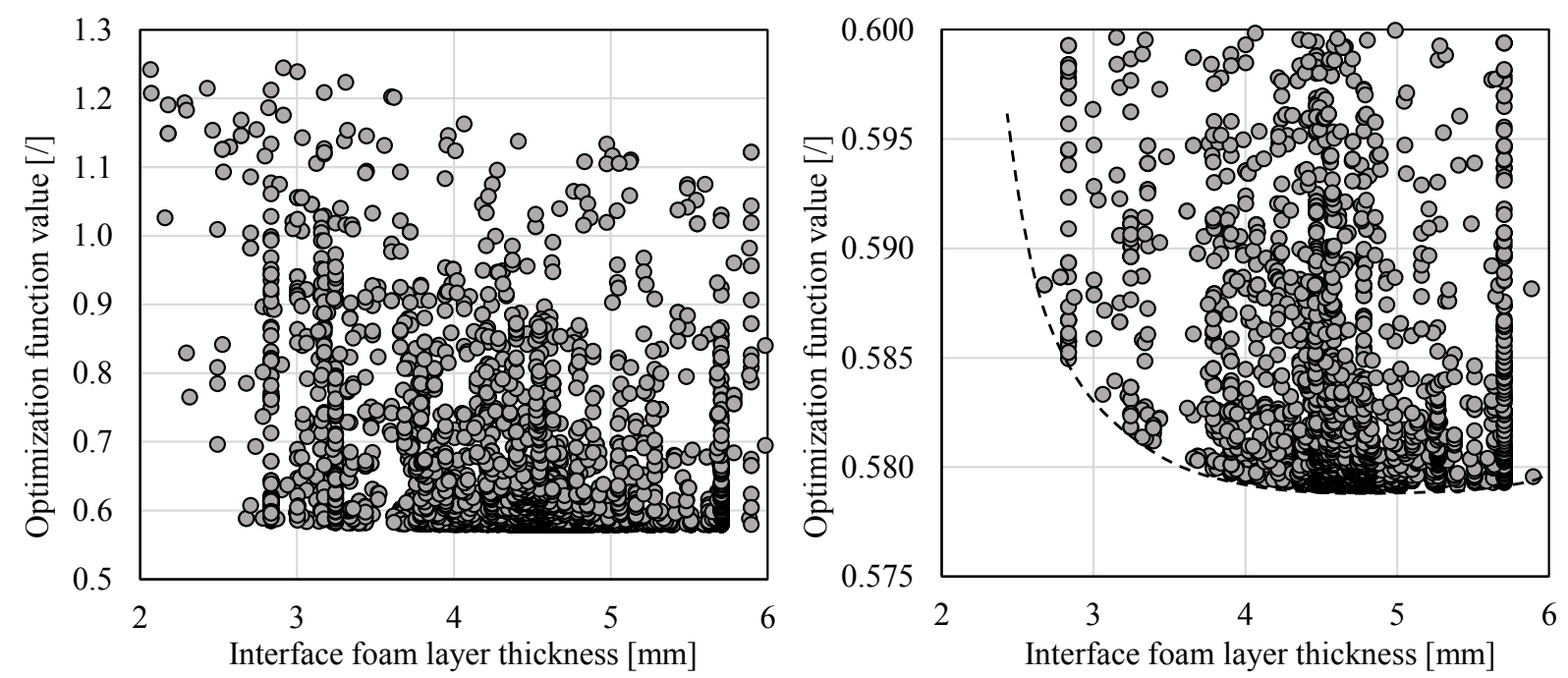

Figure 5: The optimization function value in relation to the interface foam layer thickness. 
Fig. 6 shows the effect of the interface foam thickness on the values of normalized displacement and normalized pressure slope. The foam thickness design space was divided in four discrete intervals from 2 to $6 \mathrm{~mm}$ with a width of $1 \mathrm{~mm}$ for clearer visualization.

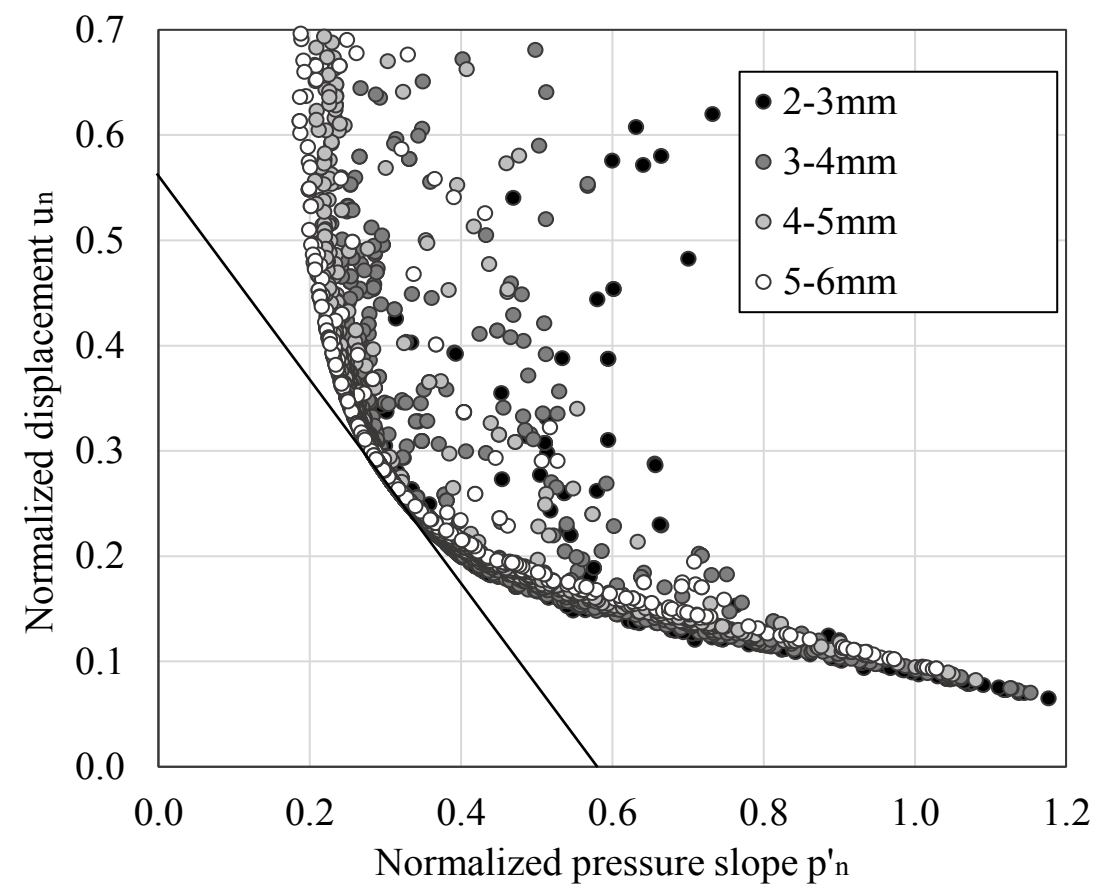

Figure 6: The solutions of the optimization problem by the interface foam layer height.

The optimal solution determined by the optimisation process is the foam layer thickness of $4.83 \mathrm{~mm}$ with the following foam material parameters $\varepsilon_{2}=0.064, \varepsilon_{3}=0.486, \sigma_{2}=59 \mathrm{kPa}$, $\sigma_{3}=116 \mathrm{kPa}$ and $\sigma_{4}=796 \mathrm{kPa}$. The deformation of the fingertip and foam for this solution is shown in Fig. 7.

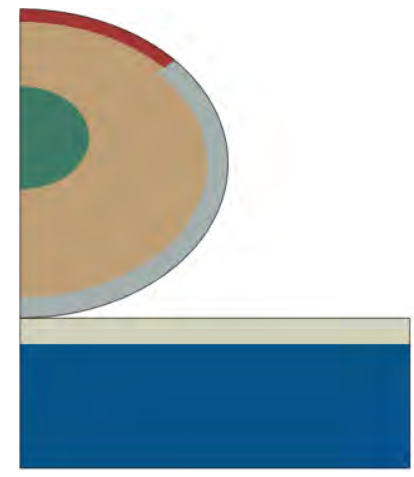

$u_{f}=0.0 \mathrm{~mm}$

$p=0.0 \mathrm{kPa}$

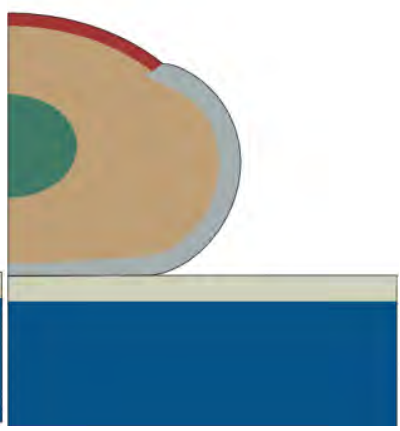

$u_{f}=2.0 \mathrm{~mm}$

$p=11.2 \mathrm{kPa}$

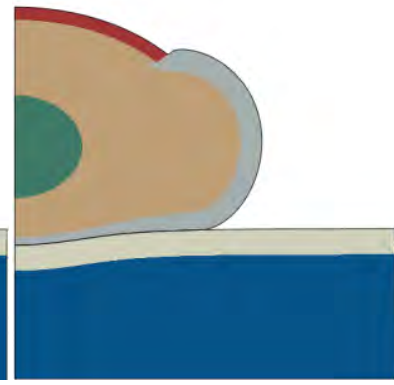

$u_{f}=3.8 \mathrm{~mm}$

$p=100.0 \mathrm{kPa}$

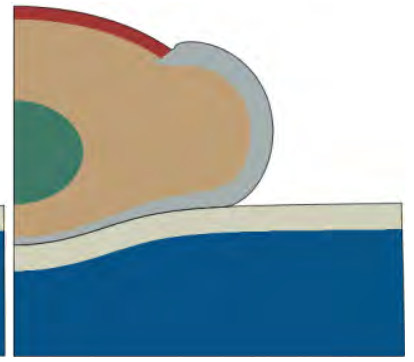

$u_{f}=5.0 \mathrm{~mm}$

$p=132.6 \mathrm{kPa}$

Figure 7: The deformation of the fingertip and tool handle at different fingertip displacements.

Response of the simulated system using optimised parameters was compared also to other product interface materials to quantify the results of the optimisation. These were the steel as quasi-rigid (Young's modulus of $210 \mathrm{GPa}$ and a Poisson ratio of 0.3) and a composite of EPDM rubber and PU foam as product's interface material proposed in one of our previous papers [30]. Material behaviour under uniaxial compression of the optimised foam parameters and thickness proposed in this paper and PU foam used in previous research can be seen in Fig. 8. 


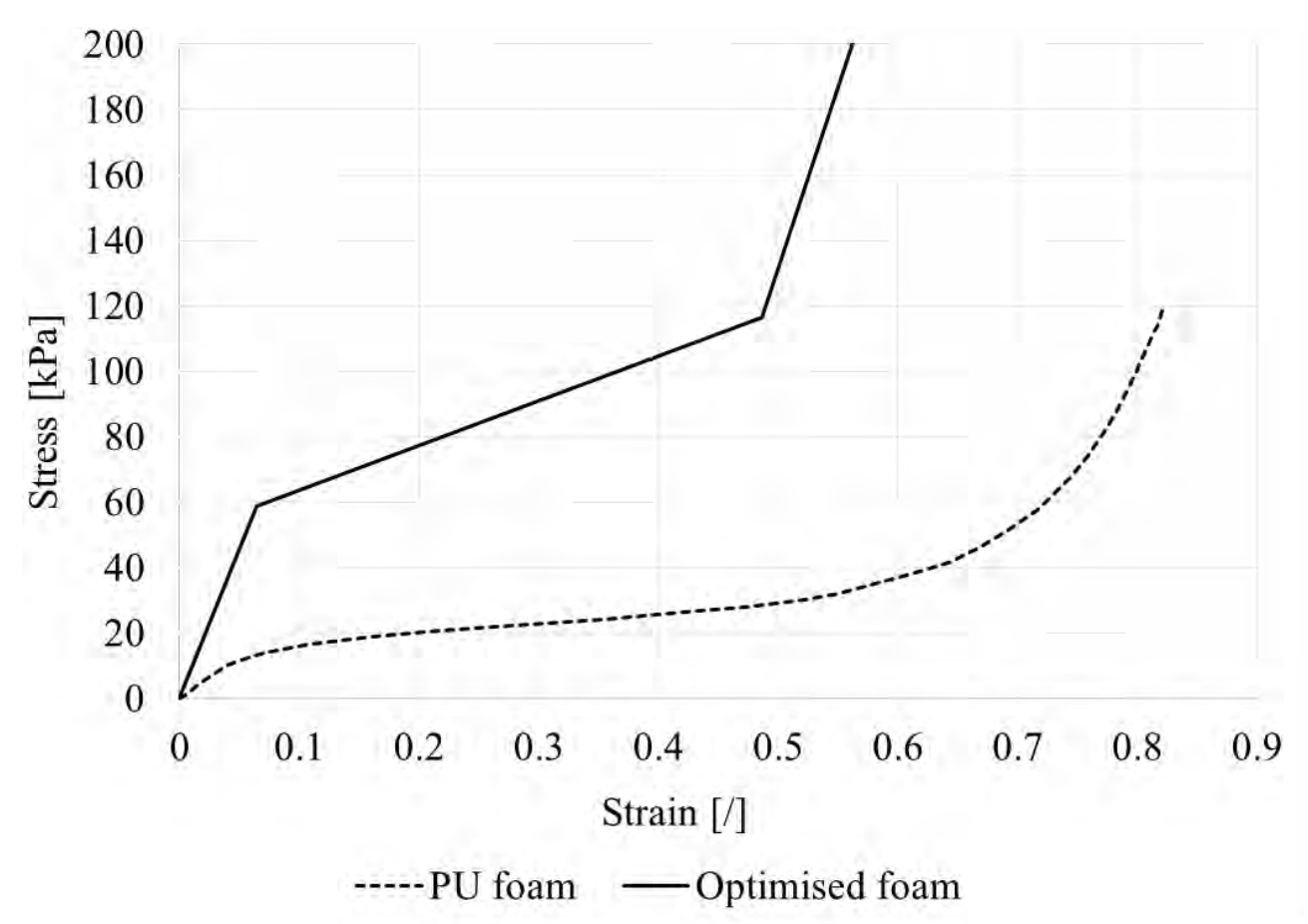

Figure 8: Stress strain responses of PU foam and optimised foam under uniaxial compression.

Since optimisation was performed in regard to contact pressure and displacement of fingertip and interface material, the results of the contact pressure in comparison to the combined vertical displacement of the fingertip and interface material were plotted (Fig. 9).

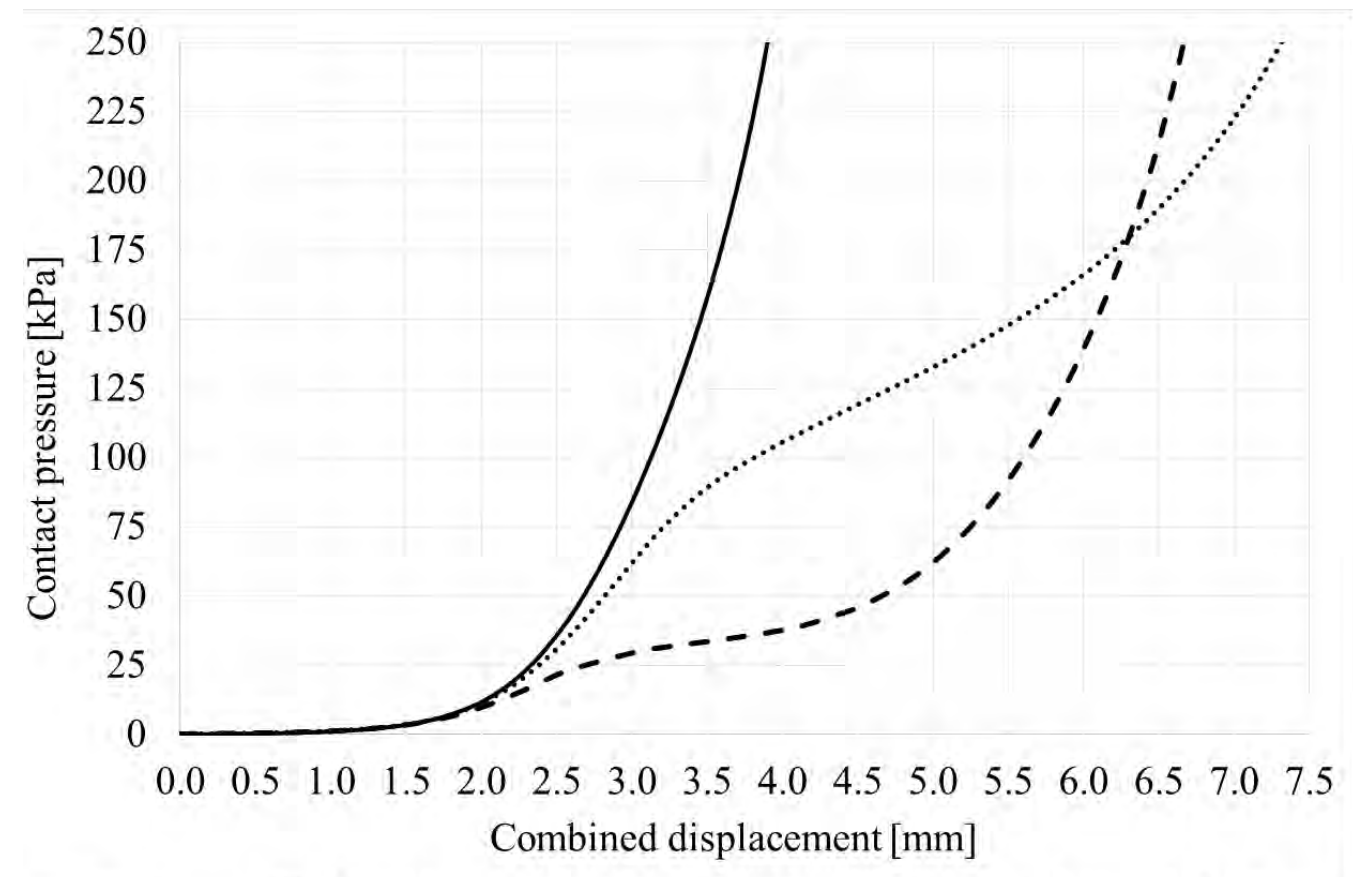

- Steel …...EPDM rubber and optimised foam - - EPDM rubber and PU foam

Figure 9: Contact pressure vs combined displacement of the fingertip and interface material.

Results show that all curves coincide to vertical displacement of about $1.7 \mathrm{~mm}$. After that point every interface material shows its unique behaviour. The steel exhibits the highest contact pressure for the given displacement. Contact pressure of $100 \mathrm{kPa}$ is reached at the vertical displacement of $3.1 \mathrm{~mm}$. The response from the interface material of EPDM rubber 
and PU foam shows reduction in contact pressure for the given vertical displacement in comparison to steel. Contact pressure of $100 \mathrm{kPa}$ is reached at the vertical displacement of 5.6 $\mathrm{mm}$. The optimized interface material of EPDM rubber and optimized foam shows contact pressure reduction at lower displacement compared to interface material of EPDM rubber and PU foam. In simulation of the optimized material the contact pressure of $100 \mathrm{kPa}$ is reached at vertical displacement of $3.85 \mathrm{~mm}$. At about $6.3 \mathrm{~mm}$ of combined vertical deformation intersection between responses of both foam composites can be observed.

\section{DISCUSSION}

It is evident from Fig. 4 that a Pareto front is formed since the normalized displacement and normalized pressure slope are in an inverse relationship. The Pareto front (black circles in Fig. 4) contains all optimization problem solutions where the improvement of one part of the optimization function is only possible if the value of the other optimization function parts is reduced. The black line in Fig. 4 represents the line where the value of the optimization function is constant and minimal. It can be seen from the figure that individuals are equally spread on both sides of the minimum and that there is only one noticeable global minimum.

The dashed line in the right diagram of Fig. 5 shows the approximated minimal value of the optimization function in dependence from the interface foam layer thickness which can be used to predict the optimal solution at a certain foam thickness selected by the user. The density of the solutions is low at higher values of the optimization function and much higher in the neighbourhood of the minima. This suggests that the convergence of the algorithm was good in terms of the population basis. Some areas with the same interface foam layer thickness are also evident in Fig. 5. They are the consequence of the mutation operator in the genetic algorithm which is used to create the next population.

The Pareto front in Fig. 6 consists from solutions with large foam thickness (white circles) at lower values of the normalized pressure slope and from solutions with small foam thickness (black circles) at higher values of the normalized pressure slope. The transition from large foam thickness to small foam thickness occurs between the normalized pressure slope of 0.3 and 0.4 . This means that if the best solution with a small normalized pressure slope would be sought the best solution would have a large foam thickness and if the best solution with a large normalized pressure slope would be sought the best solution would have a small foam thickness. Opposite relations can be made for the normalized displacement.

From Fig. 7 it is visible that in the first stages of the simulation up to the fingertip displacement of $2 \mathrm{~mm}$ only skin and the soft tissue deform, where they are in contact with the foam. As the fingertip displacement increases the deformation of the foam becomes visible. And at the contact pressure of $100 \mathrm{kPa}$ the foam starts to deform faster due to the smaller contact pressure slope.

From Fig. 8 it is clearly visible that PU foam proposed by estimation in our previous research is completely different to the optimised foam proposed in this paper. This shows that correct material parameter determination in such complex systems is necessary and possible with use of optimisation procedures as demonstrated in this paper.

Power grasps of various products can yield high contact pressures exceeding $100 \mathrm{kPa}$, which leads to ATD and CTD development. Therefore the designer of products has to consider ergonomics in order to develop sizes, shapes and interface materials, which distribute the contact pressures evenly and do not exceed the limit pressure. Hence the peak contact pressure at the fingertip center line compared to the combined vertical displacement of the fingertip and interface material was investigated and plotted in Fig. 9. Results have shown that the highest contact pressure was obtained with steel as a quasi-rigid tool-handle material. Steel is much stiffer than skin and subcutaneous tissue therefore the deformations of the steel 
as tool-handle material are negligibly small and only fingertip deformation occurs. Therefore steel as a reference interface material to evaluate other interface materials was considered. The results of both foam composites showed lower peak contact pressures due to the foam material deformation, which causes different contact pressure distribution and larger contact area. From the results it is evident that all three interface materials (steel, EPDM rubber and PU foam, EPDM rubber and optimised foam) also showed almost the same contact pressure versus vertical deformation behaviour up to a deformation of about $1.7 \mathrm{~mm}$, which is due to the deformation of the fingertip. After that both composites of EPDM rubber and PU foam and EPDM rubber and optimised foam start to deform. Due to different stress strain behaviour and different plateau levels of both composites, the diagrams have different characteristics. The lower and very prominent deformation plateau of the PU Foam shows that the interface material starts to deform at around $30 \mathrm{kPa}$ of contact pressure. According to literature contact pressure of $30 \mathrm{kPa}$ is still safe and occurs during normal handling of products in hands. Therefore deformations of the interface material at these contact pressure are not preferred as this would lower the stability of the product in hands. On the other hand the interface material using the optimised foam shows just slightly larger deformations compared to the steel for the given contact pressure. The deformation plateau of the optimised foam is reached at around $60 \mathrm{kPa}$. At this point the optimised foam starts to deform and thereby provides higher contact area and lowers the contact pressure. The intersection between response curves of both composites indicates that with the optimisation procedure a foam material with delayed densification is favourable. After the combined vertical deformation of $6.3 \mathrm{~mm}$ the composite consisting of EPDM rubber and optimised foam accounts for lower contact pressure than the composite consisting of EPDM rubber and PU foam.

\section{CONCLUSION}

According to the presented results, it can be seen that product interface material parameters cannot be easily determined. The optimisation procedure is required to establish the desired mechanical response of the simulated system. The optimisation of the interface material parameters considered that the interface material should not deform at lower contact pressures, but only when higher contact pressure was achieved, thus lowering the risk of ATD and CTD development. Using the objective function and appropriate weights the optimisation process yielded the most appropriate foam thickness and its stress-strain relationship to produce low contact pressures while maintaining high level of stability of the product grasp.

Future work should consider improving the objective function in regard of subjective responses from test users. Additionally dynamic simulations could be carried out to simulate the effect of vibration and foam damping to optimise also those parameters. Three dimensional simulations should also be performed to consider more realistic geometry of a human fingertip contact and further improve the results.

\section{ACKNOWLEDGEMENTS}

The paper was co-produced within the framework of the operation entitled "Centre of Open innovation and Research UM (CORE@UM)." The operation is co-funded by the European Regional Development Fund and conducted within the framework of the Operational Program for Strengthening Regional Development Potentials for the period 2007-2013, development priority 1: "Competitiveness of companies and research excellence", priority axis 1.1:"Encouraging competitive potential of enterprises and research excellence", contract No. 3330-13-500032.

The paper was also produced within the framework of research programme P2-063 entitled "Design of Porous Structures", which is financed by the Slovenian Research Agency "ARRS". 


\section{REFERENCES}

[1] Moore, A.; Wells, R.; Ranney, D. (1991). Quantifying exposure in occupational manual tasks with cumulative trauma disorder potential, Ergonomics, Vol. 34, No. 12, 1433-1453, doi: $\underline{10.1080 / 00140139108964888}$

[2] Bernard, B.; Nelson, N.; Estill, C. F.; Fine, L. (1998). The NIOSH review of hand-arm vibration syndrome: Vigilance is crucial, Journal of Occupational and Environmental Medicine, Vol. 40, No. 9, 780-785

[3] Youakim, S. (2009). Hand-arm vibration syndrome (HAVS), British Columbia Medical Journal, Vol. 51, No. 1, 10

[4] Rempel, D. M.; Harrison, R. J.; Barnhart, S. (1992). Work-related cumulative trauma disorders of the upper extremity, JAMA: The Journal of the American Medical Association, Vol. 267, No. 6, 838-842, doi:10.1001/jama.1992.03480060084035

[5] Punnett, L.; Wegman, D. H. (2004). Work-related musculoskeletal disorders: the epidemiologic evidence and the debate, Journal of Electromyography and Kinesiology, Vol. 14, No. 1, 13-23, doi:10.1016/j.jelekin.2003.09.015

[6] Du, S.; Zhang, A.; Yang, X.; Zhuang, Y. (2008). The study on intelligent technology integrating Ergonomics into industrial design, $9^{\text {th }}$ International Conference on Computer-Aided Industrial Design and Conceptual Design (CAID/CD 2008), 21-25, doi:10.1109/CAIDCD.2008.4730510

[7] Hogberg, D.; Backstrand, G.; Lamkull, D.; Hanson, L.; Ortengren, R. (2008). Industrial customisation of digital human modelling tools, International Journal of Services Operations and Informatics, Vol. 3, No. 1, 53-70, doi:10.1504/IJSOI.2008.017705

[8] Kaljun, J. (2014). Intelligent support for defining aesthetical, ergonomical and material properties of designed product, Technical Gazette, Vol. 21, No. 4, 835-842

[9] Garneau, C. J.; Parkinson, M. B. (2012). Optimization of product dimensions for discrete sizing applied to a tool handle, International Journal of Industrial Ergonomics, Vol. 42, No. 1, 56-64, doi:10.1016/j.ergon.2011.08.005

[10] Kong, Y.-K.; Lowe, B. D. (2005). Optimal cylindrical handle diameter for grip force tasks, International Journal of Industrial Ergonomics, Vol. 35, No. 6, 495-507, doi:10.1016/j.ergon. 2004.11.003

[11] Seo, N. J.; Armstrong, T. J. (2008). Investigation of grip force, normal force, contact area, hand size, and handle size for cylindrical handles, Human Factors, Vol. 50, No. 5, 734-744, doi: $10.1518 / 001872008 X 354192$

[12] Harih, G. (2014). Decision support system for generating ergonomic tool-handles, International Journal of Simulation Modelling, Vol. 13, No. 1, 5-15, doi:10.2507/IJSIMM13(1)1.234

[13] Clark, J. A.; Cheng, J. C. Y.; Leung, K. S. (1996). Mechanical properties of normal skin and hypertrophic scars, Burns, Vol. 22, No. 6, 443-446, doi:10.1016/0305-4179(96)00038-1

[14] Edwards, C.; Marks, R. (1995). Evaluation of biomechanical properties of human skin, Clinics in Dermatology, Vol. 13, No. 4, 375-380, doi:10.1016/0738-081X(95)00078-T

[15] Pan, L.; Zan, L.; Foster, F. S. (1998). Ultrasonic and viscoelastic properties of skin under transverse mechanical stress in vitro, Ultrasound in Medicine and Biology, Vol. 24, No. 7, 9951007, doi:10.1016/S0301-5629(98)00071-4

[16] Wan Abas, W. A. (1994). Biaxial tension test of human skin in vivo, Bio-Medical Materials and Engineering, Vol. 4, No. 7, 473-486

[17] Wilhelmi, B. J.; Blackwell, S. J.; Mancoll, J. S.; Phillips, L. G. (1998). Creep vs. stretch: a review of the viscoelastic properties of skin, Annals of Plastic Surgery, Vol. 41, No. 2, 215-219

[18] Zheng, Y.-P.; Mak, A. F. T. (1996). An ultrasound indentation system for biomechanical properties assessment of soft tissues in-vivo, IEEE Transactions on Biomedical Engineering, Vol. 43, No. 9, 912-918, doi:10.1109/10.532125

[19] Wu, J. Z.; Cutlip, R. G.; Andrew, M. E.; Dong, R. G. (2007). Simultaneous determination of the nonlinear-elastic properties of skin and subcutaneous tissue in unconfined compression tests, Skin Research and Technology, Vol. 13, No. 1, 34-42, doi:10.1111/j.1600-0846.2007.00182.x

[20] Radwin, R. G.; Armstrong, T. J.; Chaffin, D. B. (1987). Power hand tool vibration effects on grip exertions, Ergonomics, Vol. 30, No. 5, 833-855, doi:10.1080/00140138708969772 
[21] Riedel, S. (1995). Consideration of grip and push forces for the assessment of vibration exposure, Central European Journal of Public Health, Vol. 3, Supplement, 139-141

[22] Eksioglu, M. (2004). Relative optimum grip span as a function of hand anthropometry, International Journal of Industrial Ergonomics, Vol. 34, No. 1, 1-12, doi:10.1016/j.ergon. 2004.01.007

[23] Gurram, R.; Rakheja, S.; Gouw, G. J. (1995). A study of hand grip pressure distribution and EMG of finger flexor muscles under dynamic loads, Ergonomics, Vol. 38, No. 4, 684-699, doi: $10.1080 / 00140139508925140$

[24] Brand, P. W.; Hollister, A. M. (1999). Clinical mechanics of the hand, $3^{\text {rd }}$ ed., Mosby, St. Louis

[25] Aldien, Y.; Welcome, D.; Rakheja, S.; Dong, R.; Boileau, P.-E. (2005). Contact pressure distribution at hand-handle interface: role of hand forces and handle size, International Journal of Industrial Ergonomics, Vol. 35, No. 3, 267-286, doi:10.1016/j.ergon.2004.09.005

[26] Fransson-Hall, C.; Kilbom, A. (1993). Sensitivity of the hand to surface pressure, Applied Ergonomics, Vol. 24, No. 3, 181-189, doi:10.1016/0003-6870(93)90006-U

[27] Fellows, G. L.; Freivalds, A. (1991). Ergonomics evaluation of a foam rubber grip for tool handles, Applied Ergonomics, Vol. 22, No. 4, 225-230, doi:10.1016/0003-6870(91)90225-7

[28] Novak, M. (2012). Computer aided decision support in product design engineering, Technical Gazette, Vol. 19, No. 4, 743-752

[29] Vesenjak, M.; Borovinsek, M.; Fiedler, T.; Higa, Y.; Ren, Z. (2013). Structural characterisation of advanced pore morphology (APM) foam elements, Materials Letters, Vol. 110, 201-203, doi:10.1016/j.matlet.2013.08.026

[30] Harih, G.; Dolsak, B. (2014). Recommendations for tool-handle material choice based on finite element analysis, Applied Ergonomics, Vol. 45, No. 3, 577-585, doi:10.1016/j.apergo.2013. $\underline{07.014}$

[31] Dassault Systèmes (2010). Abaqus 6.10 Online Documentation. Generated: Wed April 28, 2010 at 12:31:09, Build ID: 2010_04_27-15.17.36 37133, (C) Dassault Systèmes

[32] Wu, J. Z.; Dong, R. G. (2005). Analysis of the contact interactions between fingertips and objects with different surface curvatures, Proceedings of the Institution of Mechanical Engineers, Part H: Journal of Engineering in Medicine, Vol. 219, No. 2, 89-103, doi:10.1243/095441105X9327

[33] Wu, J. Z.; Dong, R. G.; Rakheja, S.; Schopper, A. W. (2002). Simulation of mechanical responses of fingertip to dynamic loading, Medical Engineering \& Physics, Vol. 24, No. 4, 253 264, doi:10.1016/S1350-4533(02)00018-8

[34] Nidhiry, N. M.; Saravanan, R. (2014). Scheduling optimization of a flexible manufacturing system using a modified NSGA-II algorithm, Advances in Production Engineering \& Management, Vol. 9, No. 3, doi:10.14743/apem2014.3.183

[35] Mitchell, M. (1998). An introduction to genetic algorithms, MIT Press, Cambridge 